\title{
Direct Metal-Free Chemical Vapor Deposition of Graphene Films on Insulating Substrates for Micro-Supercapacitors with High Volumetric Capacitance
}

\author{
Michael Wuttke ${ }^{+[a]}$ Zhaoyang Liu ${ }^{+[a]}{ }^{[a o}$ Lu, $^{[a]}$ Akimitsu Narita, ${ }^{*[a, b]}$ and Klaus Müllen ${ }^{*[a, c]}$
}

Direct, metal-free synthesis of graphene films on insulating substrates in a controlled manner is of great importance for applications in (opto)electronic and energy storage devices. Graphene films are fabricated on fused silica substrates without metal catalyst via chemical vapor deposition (CVD), using propionic acid as a carbon source. Film-thickness is readily adjustable between 5 and $45 \mathrm{~nm}$ by changing the deposition time and flow rate of the precursor, displaying sheet resistance in the range of $0.27-1.86 \mathrm{k} \Omega \square^{-1}$. The resulting graphene films are directly integrated into micro-supercapacitors without film transfer or liquid-phase processing, and demonstrate ultrahigh operation rates up to $1000 \mathrm{Vs}^{-1}$. Moreover, these films exhibit excellent rate capabilities, achieving a high volumetric capacitance of $131 \mathrm{~F} \mathrm{~cm}^{-3}$ at $10 \mathrm{mV} \mathrm{s}^{-1}$.

\section{Introduction}

With the ever-increasing demand for portable and miniaturized electronics, lightweight, flexible, and highly efficient microscale energy storage systems are in high demand. ${ }^{[1-3]}$ Among them, micro-supercapacitors (MSCs) are particularly promising since they combine high power density with fast charge/ discharge rates and long cycle lives. ${ }^{[4-6]}$ Graphene is attractive as the active component of efficient MSCs because of its superior electrical conductivity, high specific surface area, and outstanding intrinsic double-layer capacitance. However, fabrication of high-performance MSCs based on graphene films has remained elusive, because they tend to have low volumetric capacitance. ${ }^{[7,8]}$ The limited performance can be attributed largely to the lack of proper thickness control on suitable substrates over large areas ${ }^{[9,10]}$ and/or to relatively poor electronic properties of graphene samples used for MSCs. ${ }^{[7,11,12]}$

[a] M. Wuttke, ${ }^{+}$Dr. Z. Liu, ${ }^{+}$Dr. H. Lu, Dr. A. Narita, Prof. Dr. K. Müllen Max Planck Institute for Polymer Research

Ackermannweg 10, D-55128 Mainz, Germany

E-mail: narita@mpip-mainz-mpg.de

[b] Dr. A. Narita muellen@mpip-mainz.mpg.de

Organic and Carbon Nanomaterials Unit

Okinawa Institute of Science and Technology Graduate University

1919-1 Tancha, Onna-son, Kunigami, Okinawa, 904-0495, Japan

[c] Prof. Dr. K. Müllen

Institute of Physical Chemistry

Johannes Gutenberg-University Mainz

Duesbergweg 10-14, 55128 Mainz, Germany

$\left.{ }^{[+}\right]$These authors contributed equally to this work.

Supporting information for this article is available on the WWW under https://doi.org/10.1002/batt.201900087

- This publication is part of a joint Special Issue with ChemSusChem focusing on "2D Energy Storage Materials".

of (c) 2019 The Authors. Published by Wiley-VCH Verlag GmbH \& Co. KGaA This is an open access article under the terms of the Creative Commons Attribution License, which permits use, distribution and reproduction in any medium, provided the original work is properly cited.
Thickness and electronic properties of graphene films are largely determined by the fabrication method. Graphene samples used for the MSC applications have typically been prepared using electrochemical exfoliation of graphite, ${ }_{1}^{[13]}$ chemical reduction of graphene oxide $(\mathrm{rGO}),{ }^{[14]}$ and chemical vapor deposition (CVD). ${ }^{[15]}$ Films of electrochemically exfoliated graphene $(E G)$ are prepared through filtration of its dispersion in isopropanol with membrane filters, followed by transfer onto desired substrates. Film thickness can be controlled by changing the concentration and/or amount of the dispersion. However, MSCs prepared with such EG films exhibit poor performance, presumably due to the relatively low electronic conductivity of the films, which compromises the electrical double-layer capacitance. ${ }^{[11,16,17]}$ Performance can be improved by introducing additional pseudocapacitive conducting polymers, such as poly(3,4-ethylenedioxy thiophene):poly(styrenesulfonate) (PEDOT: PSS) and polyaniline (PANI), but volumetric capacitance remains below $100 \mathrm{Fcm}^{-3}$. $[8,9]$ MSCs based on $\mathrm{rGO}$ films generally display even worse device performance compared with MSCs utilizing EG films, with volumetric capacitances below $20 \mathrm{~F} \mathrm{~cm}^{-3} \cdot{ }^{[11,17]}$ In contrast, graphene films prepared by the CVD method have higher quality with fewer structural defects and better electronic properties, which are advantageous for fabrication of high-performance MSCs.

CVD graphene films are mainly prepared through metalcatalyzed processes using copper foils as substrates, which can easily be scaled up to large areas or incorporated into roll-toroll processes. ${ }^{[18,19]}$ Nevertheless, it is difficult to cleanly transfer the films from the metal surface to desired substrates (e.g., dielectrics, insulators) for further applications. ${ }^{[20]}$ Post-growth film transfer is carried out by etching the metal catalysts while using a polymer support, which inevitably compromises the quality of the graphene films, inducing, e.g., breaks, wrinkles, and contaminants of the surface with polymer residues. To this end, many attempts have been made to grow graphene films directly on insulating substrates without metal catalysts, for example, by applying techniques such as atmospheric pressure 
CVD (APCVD) $)^{[21-23]}$ and plasma-enhanced CVD (PECVD). ${ }^{[24-27]}$ However, transfer-free routes by direct CVD growth still suffer multiple drawbacks, including inhomogeneous nucleation at the macroscopic level, which results in small graphene domain sizes and poor film qualities. Moreover, the applications of graphene films produced by direct growth approaches have been limited to transparent conductive electrodes. Fabrication of high-quality graphene films with tunable thickness using the direct CVD approach, and its application to energy storage devices such as MSCs has remained elusive.

Here we demonstrate direct growth of CVD graphene on insulating fused silica substrates, using propionic acid as a precursor, and its integration into all-solid-state MSCs. By optimizing the low-pressure CVD (LPCVD) process, we were able to tune the thickness, optical transparencies, and electrical conductivities of the deposited graphene. We further fabricated MSCs using the as-grown graphene films on fused silica, which allowed operations at an ultrahigh rate up to $1000 \mathrm{Vs}^{-1}$, three orders of magnitude higher than conventional sandwichstructured electric double-layer capacitors. The device moreover exhibited an outstanding volumetric capacitance of $131 \mathrm{~F} \mathrm{~cm}^{-3}$ at $10 \mathrm{mV} \mathrm{s}^{-1}$, and an excellent rate capability of $27 \%$, when operated from 10 to $10^{5} \mathrm{mV} \mathrm{s}^{-1}$.

\section{Results and Discussion}

Whereas molecules like methane have been used as precursors for direct growth of graphene on fused silica, we chose propionic acid, hypothesizing that a larger molecule with a lower decomposition temperature could be advantageous (decomposition temperature; methane: $1200^{\circ} \mathrm{C}_{1}^{[28]}$ propionic acid: $\left.496{ }^{\circ} \mathrm{C}^{[29]}\right)$. The use of a carboxyl group appeared promising in view of previous density functional theory (DFT) studies, suggesting that the presence of oxygen-containing functional groups could enhance adsorption of hydrocarbons onto fused silica. ${ }^{[30]}$ Fused silica was selected as the substrate material due to its low cost and high softening point, which allow high-temperature growth on the substrate surface. Graphene growth was achieved using a tube furnace with a diameter of $5 \mathrm{~cm}$ at a fixed temperature of $1100^{\circ} \mathrm{C}$. The fluid precursor was evaporated at a room temperature from a glass vial, connected by a valve. The vial containing the propionic acid was placed in a water bath to maintain the temperature during the evaporation. The evaporation of the precursor was controlled by adjusting the increase of the overall system pressure $(\Delta p)$ after opening the valve. Raman spectra of the resulting graphene samples showed $D, G$, and $2 D$ bands typical for turbostratic multilayer graphene. ${ }^{[31]}$ The $G$ band is induced by the first-order scattering of the $\mathrm{sp}^{2}$ carbon, the $\mathrm{D}$ band by lattice defects and boundaries, and the $2 \mathrm{D}$ band results from second-order scattering of the $D$ band. ${ }^{[32]}$ The $2 \mathrm{D}$ band of our as-grown graphene sample is observed at $2714 \mathrm{~cm}^{-1}$, without any shoulder, which is distinct from the $2 \mathrm{D}$ band of highly oriented pyrolytic graphite (HOPG) that is upshifted to $2739 \mathrm{~cm}^{-1}$ with a shoulder appearing at $2704 \mathrm{~cm}^{-1}$ (Figure $1 \mathrm{a}) .{ }^{[33]}$ The $2 \mathrm{D}$ band of our sample also shows a broadening
Table 1. Growth time and pressure increase $(\Delta p)$ during preparation of graphene samples and their sheet-resistance, transmittance and $I_{D} / I_{G}$ ratio. Sample Growth time (h) $\Delta p$ (mbar)

Transmission $_{550 \mathrm{~nm}}(\%)$ Sheet-resistance $\left(\mathrm{k} \Omega \square^{-1}\right)$ $I_{D} / I_{G}$ ratio

Thickness (nm)

\begin{tabular}{llllll}
1 & 2 & 3 & 4 & 5 & 6 \\
2 & 2 & 2 & 2 & 3 & 3 \\
0.13 & 0.06 & 0.04 & 0.02 & 0.02 & 0.01 \\
22.3 & 45.6 & 55.6 & 86.1 & 47.2 & 80.4 \\
0.27 & 0.47 & 0.69 & 1.86 & 0.60 & 1.41 \\
0.62 & 0.53 & 0.52 & 0.72 & 0.58 & 0.50 \\
45 & 30 & - & 5 & - & - \\
\hline
\end{tabular}

Table 2. Comparison of $I_{\mathrm{D}} / I_{\mathrm{G}}$ and $I_{2 \mathrm{D}} / I_{\mathrm{G}}$ of the as-grown sample 6 with graphene grown on copper, EG and $\mathrm{rGO}$ calculated from Raman spectrum (Figure S2)

\begin{tabular}{|lllll|} 
& Sample 6 & Graphene grown on copper & EG & rGO \\
\hline$I_{\mathrm{D}} / I_{\mathrm{G}}$ & 0.50 & $/$ & 0.41 & 0.95 \\
$I_{2 \mathrm{D}} / I_{\mathrm{G}}$ & 0.48 & 1.63 & 0.29 & $/$ \\
\hline
\end{tabular}

presumably due to the relaxation of the double resonance Raman selection rules originating from the random orientation of the graphene layers, suggesting the existence of turbostratic multilayer graphene. ${ }^{[31]}$ Graphene quality could be greatly improved, based on the ratio of the $D$ and $G$ bands in Raman spectra $\left(I_{\mathrm{D}} / I_{\mathrm{G}}\right){ }^{[34]}$ by increasing deposition time from $2 \mathrm{~h}$ to $3 \mathrm{~h}$ and by decreasing the amount of evaporated propionic acid by reducing the pressure increase (Table 1 ). Comparing the $I_{\mathrm{D}} / I_{\mathrm{G}}$ and $I_{2 \mathrm{D}} / I_{\mathrm{G}}$ ratios of sample 6 to graphene grown on copper substrates, $\mathrm{EG}^{[9]}$ and $\mathrm{rGO}$ (Table 2 ), it can clearly be seen that graphene grown on copper still owns the superior quality. However, the $I_{\mathrm{D}} / I_{\mathrm{G}}$ and $I_{2 \mathrm{D}} / I_{\mathrm{G}}$ ratios of sample 6 is comparable to that of EG and superior to $\mathrm{rGO}$, indicating the relatively high quality of graphene prepared by the current method.

Investigation of sample 6 by atomic force microscopy (AFM) revealed the growth of multi-layer graphene islands (Figure 1c) with an average height of ca. $4.1 \mathrm{~nm}$, corresponding to approximately 11 layers, based on a layer thickness of $0.37 \mathrm{~nm} .{ }^{[35]}$ UV-Vis transmission spectra revealed transmission of $80.4 \%$ at $550 \mathrm{~nm}$, which corresponds to 8.5 graphene layers, based on an absorbance of $2.3 \%$ per layer. ${ }^{[36]}$

The discrepancy in the number of calculated layers from AFM images and transmission spectra can be attributed to the growth of multilayer graphene islands, which can be seen in the AFM image and the optical microscope image (Figure 1cd). Transmission spectroscopy and four-probe measurements of graphene films grown directly on fused silica (Figure 1b) show a direct correlation between the number of layers and the sheet resistance of the samples. Sheet resistances could be tailored within a range from $0.27-1.86 \mathrm{k} \Omega \square^{-1}$ by adjusting $\Delta p$. The thinnest graphene film grown by the current method exhibits a transmittance of $86.1 \%$, corresponding to $\sim 6$ layers, and an average sheet resistance of $1.86 \mathrm{k} \Omega \square^{-1}$, whereas the thickest film exhibits a transmittance of $22.3 \%$, corresponding to $\sim 34$ layers, and an average sheet resistance of $0.27 \mathrm{k} \Omega \square^{-1}$.

The graphene film was further characterized by X-ray Photoelectron Spectroscopy (XPS) verifying that no metal species exist in the resulting samples. The survey spectrum (Figure 2) displays predominant $C$ 1s peaks from graphene, together with additional (O 1s, Si 2s, Si 2p) emissions from the 
a

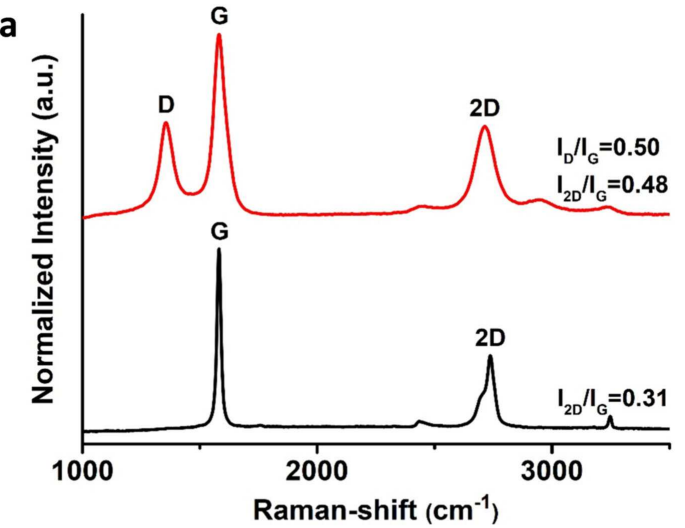

c

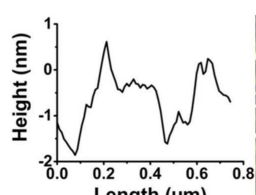

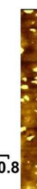

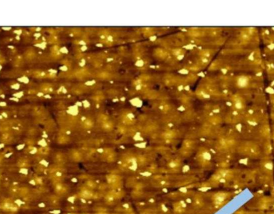

(nm)

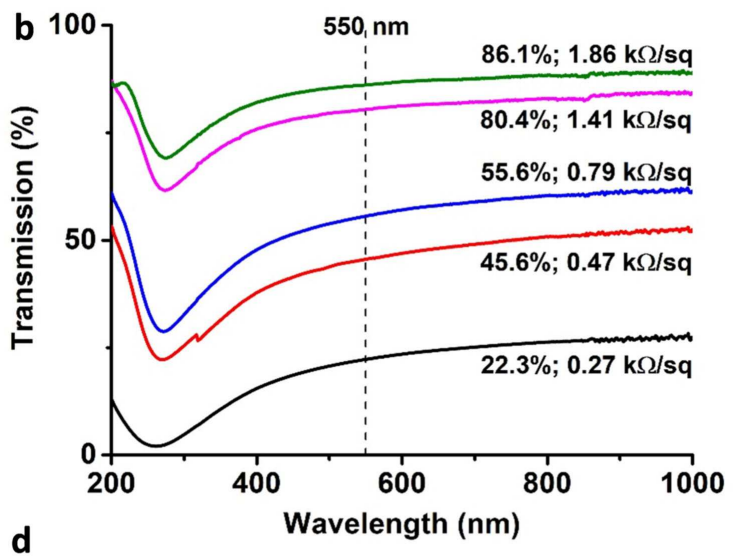

\section{5}

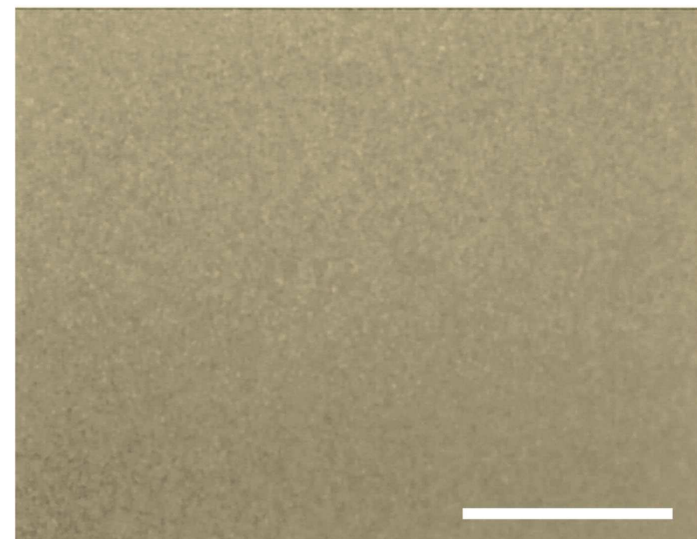

Figure 1. (a) Raman spectra of a graphene film grown directly on fused silica (red line; Sample 6 in Table 1) and HOPG (black line) (b) UV-Vis transmission spectra of graphene samples 1-4 and 6 (see Table 1) as grown on fused silica, displaying transmittance at $550 \mathrm{~nm}$ and sheet resistance. (c) AFM height image of the as-grown graphene of sample 6. Scale bar: $1 \mu \mathrm{m}$; Inset: Height profile along the blue line. Scale of height is nm. (d) Optical microscope image of the graphene film of sample 6. Scale bar: $20 \mu \mathrm{m}$; Magnification: 100x.
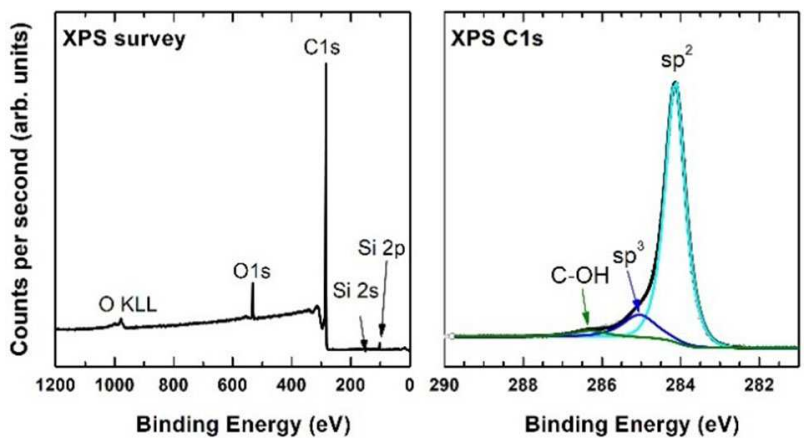

Figure 2. (left) XPS survey spectrum of sample 1. (right) Fitted C1s spectra of sample 1 showing sp ${ }^{2}$ Carbon at $284.1 \mathrm{eV}$ (cyan line), $\mathrm{sp}^{3}$ Carbon at $285 \mathrm{eV}$ (blue line) and $\mathrm{C}-\mathrm{OH}$ at $286.3 \mathrm{eV}$ (green line).

fused silica substrate. No trace of metal species can be detected, which is further verified by detailed XP spectra in the corresponding binding energy range (Figure S1). The highresolution $C$ 1s spectrum is self-fitted by individual emission peaks at binding energies of 284.1, 285 and $286.3 \mathrm{eV}$, which are assigned to $\mathrm{sp}^{2}$ carbon (cyan), $\mathrm{sp}^{3}$ carbon (blue) and $\mathrm{C}-\mathrm{OH}$ (green) bonds, respectively (Figure 2$)^{[37-39]}$ The ratio of $\mathrm{sp}^{2}$ and $\mathrm{sp}^{3}$ carbon has been determined by further quantitative analysis from the $\mathrm{C} 1 \mathrm{~s}$-spectrum. The resulting ratios of $85.5 \%$ $\mathrm{sp}^{2}, 11.5 \% \mathrm{sp}^{3}$ and only $3.1 \% \mathrm{C}-\mathrm{OH}$ indicate the high quality of the as-grown graphene films (Table S1).

Our graphene samples grown directly on fused silica substrates, with sheet resistances of $0.27-1.86 \Omega \square^{-1}$, are still inferior to bi-layer graphene grown by CVD on SiC substrates, with sheet resistances of $150 \Omega \square^{-1},{ }^{[0]}$ or single-layer graphene grown on metals, especially on Cu substrates, which show an even lower sheet resistance of $125 \Omega \square^{-1}$ with $97.4 \%$ optical transmittance. ${ }^{[19]}$ Nevertheless, graphene samples with approximately $80 \%$ transmittance produced by the current method exhibited a sheet resistance of ca. $1.4 \mathrm{k} \Omega \square^{-1}$, which is superior to those of rGO films on glass ( $82 \%$ transparency; $\left.8 \mathrm{k} \Omega \square^{-1}\right)^{[41]}$ and graphene films grown directly on fused silica using PECVD (77-82\% transparency; $2.5-4.0 \mathrm{k} \Omega \square^{-1}$ ). ${ }^{[26,42]}$ They are comparable to those of graphene produced by APCVD on fused silica (80\% transparency; $\left.1.5-2.0 \mathrm{k} \Omega \square^{-1}\right)^{[21,22]}$ and graphene grown by liquid precursor-based methods $(83.5 \%$ transparency; $\left.1.3 \mathrm{k} \Omega \square^{-1}\right) .^{[43]}$

The relatively high quality of graphene films from direct CVD growth in the current study prompted us to examine their applicability to MSC devices. Further, the tunable film thickness achievable by our method offers an effective way to optimize MSC performance. The resulting graphene samples also displayed smooth surfaces (Figure 1d), which can promote 
intimate contact between graphene electrodes and current collectors. Thus, we prepared all-solid-state MSCs based on graphene films with different thickness, i.e. $5 \mathrm{~nm}$ (sample 4), $30 \mathrm{~nm}$ (sample 2) and $40 \mathrm{~nm}$ (sample 1). MSCs were fabricated on fused silica substrates without the need of film transfer as illustrated in Figure 3a. Compared with MSC fabrication using EG and rGO, the current method avoids exfoliation and liquidphase processing, which damage the graphene film.

To evaluate performance of MSCs fabricated by our method, cyclic voltammetry (CV) was performed at different scan rates ranging from 10 to $10^{6} \mathrm{mV} \mathrm{s}^{-1}$ (Figure $3 \mathrm{~b}-\mathrm{g}$ ). All CV curves revealed quasi-rectangular shapes from 0 to $1 \mathrm{~V}$ at scan rates of 10 to $10^{4} \mathrm{mV} \mathrm{s}^{-1}$. This indicates significant electrical double-layer capacitance from graphene because of the good electronic characteristics, i.e., relatively low sheet resistance ranging from $0.27 \mathrm{k} \Omega \square^{-1}$ and $0.47 \mathrm{k} \Omega \square^{-1}$ to $1.86 \mathrm{k} \Omega \square^{-1}$. Volumetric capacitances at different scan rates were calculated as previously reported for each sample with different film thickness and compared as displayed in Figure $3 h \cdot{ }^{[7-9]}$ Remarkably, for a 5-nm graphene film, a maximum areal capacitance of $66 \mu \mathrm{F} \mathrm{cm}^{-2}$ and a volumetric capacitance of $131 \mathrm{~F} \mathrm{~cm}^{-3}$ were delivered at a scan rate of $10 \mathrm{mV} \mathrm{s}^{-1}$. This outperformed most other MSCs based on graphene or porous carbon materials, with volumetric capacitances ranging from 1.3 to $17.9 \mathrm{~F} \mathrm{~cm}^{-3} \cdot{ }^{[7,11,17,44]}$ When comparing the three graphene electrodes (Fig. $3 \mathrm{~h}$ ), not surprisingly, volumetric capacitance decreased with increasing thickness. Such thickness-dependent behavior can be partially attributed to the limited interlayer conductivity of graphene films. Namely, thinner films can transport whole charges more efficiently to the gold current collector. The volumetric capacitance remained $35 \mathrm{~F} \mathrm{~cm}^{-3}$ at a high scan rate of $10^{6} \mathrm{mV} \mathrm{s}^{-1}$, showing a capacitance retention of $27 \%$. Similarly, for $30-\mathrm{nm}$ and $45-\mathrm{nm}$ films, capacitance retention of $23 \%$ and $26 \%$ was demonstrated. These MSCs all allowed charge/discharge operations at ultrahigh rates up to $10^{6} \mathrm{mV} \mathrm{s}^{-1}$ while maintaining excellent capacitance. This is three orders of magnitude higher than conventional sandwichstructured electric double-layer capacitors and most reported high-power microdevices. ${ }^{[7]}$ Also, dependence of the discharge current on the scan rate was linear up to at least $10^{5} \mathrm{mV} \mathrm{s}^{-1}$ (Figure 3i). Such excellent rate capability can be attributed to the fast ion diffusion and charge storage behavior, which preserved more than $20 \%$ volumetric capacitance at high operating speeds. These results indicate that the direct CVD approach for graphene film production is promising for future powerful MSC applications.

\section{Conclusions}

In summary, we have developed a reliable new approach for direct graphene film deposition on fused silica using LPCVD. Layer number and sheet resistance can be controlled by adjusting growth conditions. Graphene films directly grown on fused silica exhibit notable optical and electronic properties, with a transparency from 86 to $22 \%$ at $550 \mathrm{~nm}$ and corresponding sheet resistances from $1.86 \mathrm{k} \Omega \square^{-1}$ to $0.27 \mathrm{k} \Omega \square^{-1}$. With graphene films synthesized under such carefully controlled conditions, we further fabricated MSCs employing the as-grown graphene films on fused silica substrates. The MSCs all allowed for operations at ultrahigh rates up to $1000 \mathrm{Vs}^{-1}$, which is three orders of magnitude higher than conventional sandwich-structured electric doublelayer capacitors. High performance in regard to volumetric capacitance was delivered, with excellent rate capability. Such direct deposition of graphene films on desired substrates is crucial for future on-chip MSC devices, to avoid catalyst waste, fracturing of the graphene, and contamination during the transfer process. Although nucleation and growth mechanisms of graphene films on insulating substrates have yet to be addressed, further enhancement of the properties of graphene films and MSC performance can be expected by optimizing the
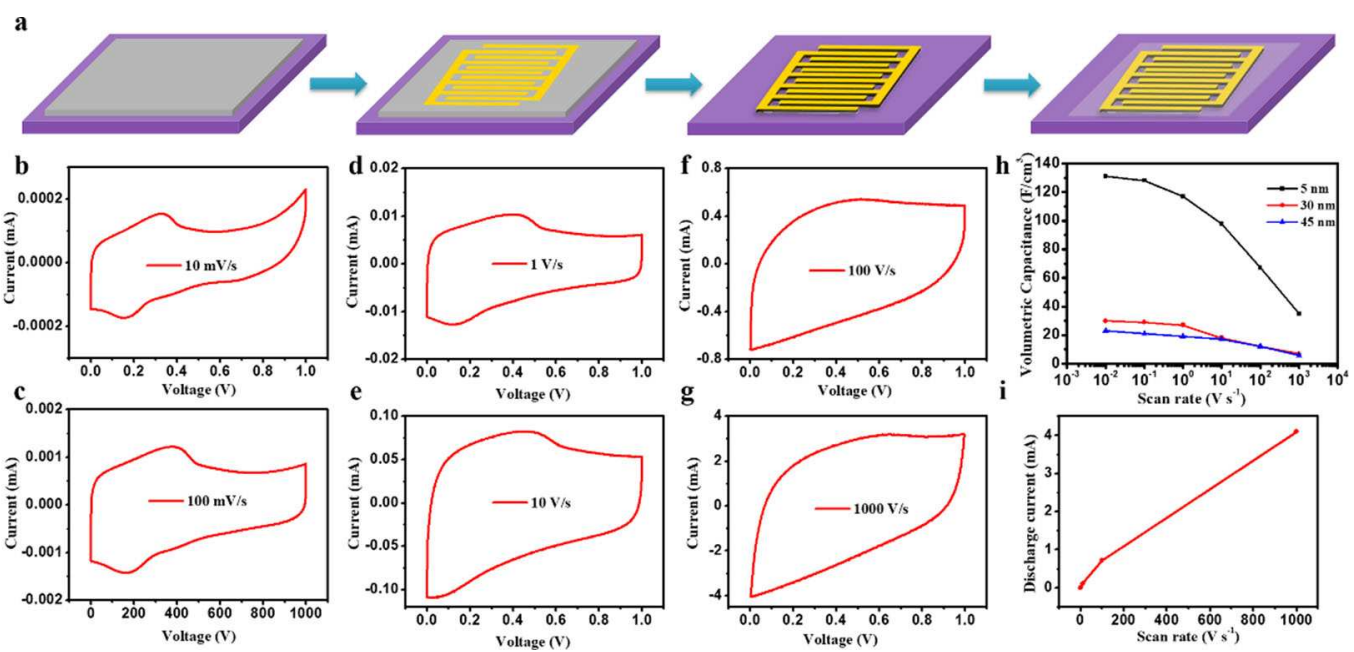

Figure 3. a) Schematic illustration of fabrication of MSC devices using CVD grown graphene films, b-g) CV curves of MSC based on sample 4 with scan rates ranging from 10 to $10^{6} \mathrm{mV} \mathrm{s}^{-1}, \mathrm{~h}$ ) evolution of volumetric capacitances versus scan rates from graphene electrodes with different thickness, and i) plot of the discharge current as a function of the scan rate. Linear dependence is observed up to $100 \mathrm{~V} \mathrm{~s}^{-1}$. 
selection of precursor molecules and substrates, to create defined porous graphene structures suitable for MSCs.

\section{Experimental Section}

Growth of graphene: A quartz tube with an inner diameter of $4.5 \mathrm{~cm}$ was fixed in a single-zone electric furnace and heated to the desired temperature. Fused silica substrates were cleaned by sonication in dichloromethane for $10 \mathrm{~min}$ and then in deionized water (DI-water) for another $10 \mathrm{~min}$. After cleaning, substrates were transferred to a horizontal quartz tube $(5 \mathrm{~cm}$ diameter) placed inside a single-zone, high-temperature furnace. The system was evacuated and flushed with $10 \mathrm{sccm}$ hydrogen for $5 \mathrm{~min}$ before starting to ramp the temperature. After reaching the desired temperature, graphene growth was initiated by opening the vial containing propionic acid. The propionic acid flow was controlled via the pressure increase in the system $(\Delta p)$ by adjusting the opening of the vial. The typical pressure increase was between $0.02-0.13 \mathrm{mbar} \pm 0.01 \mathrm{mbar}$.

Fabrication of MSC: The graphene film was directly employed as the electrode material for MSCs without any additional treatment, followed by well-established lithography techniques to achieve interdigital microelectrode patterns through the deposition of gold as current collectors. ${ }^{[8]}$ Then, an oxygen plasma was applied to remove excess graphene between the interdigital microelectrodes. Finally, a polymer gel electrolyte $\mathrm{H}_{2} \mathrm{SO}_{4}$ /polyvinyl alcohol $\left(\mathrm{H}_{2} \mathrm{SO}_{4} /\right.$ PVA) was drop-cast onto the interdigital electrodes and allowed to solidify overnight, yielding the in-plane structured MSCs.

\section{Acknowledgements}

M.W. and Z.L. contributed equally to this work. This work was supported by the Max Planck Society and the European Union's Horizon 2020 research and innovation program under grant agreement number 785219 (Graphene Flagship Core 2). We thank Steven D. Aird for editing the manuscript.

\section{Conflict of Interest}

The authors declare no conflict of interest.

Keywords: direct chemical vapor deposition - graphene volumetric capacitance · micro-supercapacitors · energy storage

[1] L. Wen, F. Li, H.-M. Cheng, Adv. Mater. 2016, 28, 4306-4337.

[2] C. Liu, F. Li, L.-P. Ma, H.-M. Cheng, Adv. Mater. 2010, 22, E28-E62.

[3] D. Qi, Y. Liu, Z. Liu, L. Zhang, X. Chen, Adv. Mater. 2017, 29, 1602802.

[4] M. Beidaghi, Y. Gogotsi, Energy Environ. Sci. 2014, 7, 867-884.

[5] N. A. Kyeremateng, T. Brousse, D. Pech, Nat. Nanotechnol. 2016, $12,7$.

[6] Z.-S. Wu, X. Feng, H.-M. Cheng, Natl. Sci. Rev. 2013, 1, 277-292.

[7] Z. Wu, K. Parvez, X. Feng, K. Müllen, Nat. Commun. 2013, 4, 2487.

[8] Z. Liu, S. Liu, R. Dong, S. Yang, H. Lu, A. Narita, X. Feng, K. Müllen, Small 2017, 13, 1603388.

[9] Z. Liu, Z.-S. Wu, S. Yang, R. Dong, X. Feng, K. Müllen, Adv. Mater. 2016, $28,2217-2222$.
[10] Z.-S. Wu, K. Parvez, S. Li, S. Yang, Z. Liu, S. Liu, X. Feng, K. Müllen, Adv. Mater. 2015, 27, 4054-4061.

[11] M. F. El-Kady, R. B. Kaner, Nat. Commun. 2013, 4, 1475.

[12] M. F. El-Kady, V. Strong, S. Dubin, R. B. Kaner, Science 2012, 335, 13261330.

[13] M. J. McAllister, J. L. Li, D. H. Adamson, H. C. Schniepp, A. A. Abdala, J. Liu, M. Herrera-Alonso, D. L. Milius, R. Car, R. K. Prud'homme, Chem Mater. 2007, 19, 4396-4404.

[14] X. Cao, D. Qi, S. Yin, J. Bu, F. Li, C. F. Goh, S. Zhang, X. Chen, Adv. Mater 2013, 25, 2957-2962.

[15] A. Reina, X. Jia, J. Ho, D. Nezich, H. Son, V. Bulovic, M. S. Dresselhaus, J. Kong, Nano Lett. 2009, 9, 30-35.

[16] M. F. El-Kady, Y. Shao, R. B. Kaner, Nat. Rev. Mater. 2016, 1, 16033.

[17] W. Gao, N. Singh, L. Song, Z. Liu, A. L. M. Reddy, L. Ci, R. Vajtai, Q. Zhang, B. Wei, P. M. Ajayan, Nat. Nanotechnol. 2011, 6, 496.

[18] X. Li, W. Cai, J. An, S. Kim, J. Nah, D. Yang, R. Piner, A. Velamakanni, I. Jung, E. Tutuc, Science 2009, 324, 1312-1314.

[19] S. Bae, H. Kim, Y. Lee, X. Xu, J.-S. Park, Y. Zheng, J. Balakrishnan, T. Lei, H. Ri Kim, Y. II Song, Nat. Nanotechnol. 2010, 5, 574-578.

[20] J. W. Suk, A. Kitt, C. W. Magnuson, Y. Hao, S. Ahmed, J. An, A. K. Swan, B. B. Goldberg, R. S. Ruoff, ACS Nano 2011, 5, 6916-6924.

[21] J. Sun, Y. Chen, M. K. Priydarshi, Z. Chen, A. Bachmatiuk, Z. Zou, Z. Chen, X. Song, Y. Gao, M. H. Rümmeli, Nano Lett. 2015, 15, 5846-5854.

[22] H. Bi, S. Sun, F. Huang, X. Xie, M. Jiang, J. Mater. Chem. 2012, 22, 411416.

[23] Y. Chen, J. Sun, J. Gao, F. Du, Q. Han, Y. Nie, Z. Chen, A. Bachmatiuk, M. K. Priydarshi, D. Ma, Adv. Mater. 2015, 27, 7839-7846.

[24] J. Sun, Y. Zhang, Z. Liu, ChemNanoMat 2016, 2, 9-18.

[25] D. Wei, Y. Lu, C. Han, T. Niu, W. Chen, A. T. S. Wee, Angew. Chem. Int. Ed. 2013, 52, 14121-14126; Angew. Chem. 2013, 125, 14371-14376.

[26] L. Zhang, Z. Shi, Y. Wang, R. Yang, D. Shi, G. Zhang, Nano Res. 2011, 4, 315-321.

[27] J. Sun, Y. Chen, X. Cai, B. Ma, Z. Chen, M. K. Priydarshi, K. Chen, T. Gao, X. Song, Q. Ji, Nano Res. 2015, 8, 3496-3504.

[28] H. F. Abbas, W. M. A. Wan Daud, Int. J. Hydrogen Energy 2010, 35, 1160 1190.

[29] P. G. Blake, K. J. Hole, J. Chem. Soc. B 1966, 577-579.

[30] B. Liu, D.-M. Tang, C. Sun, C. Liu, W. Ren, F. Li, W.-J. Yu, L.-C. Yin, L. Zhang, C. Jiang, J. Am. Chem. Soc. 2011, 133, 197-199.

[31] L. M. Malard, M. A. Pimenta, G. Dresselhaus, M. S. Dresselhaus, Phys. Rep. 2009, 473, 51-87.

[32] A. C. Ferrari, D. M. Basko, Nat. Nanotechnol. 2013, 8, 235-246.

[33] L. G. Cançado, A. Reina, J. Kong, M. S. Dresselhaus, Phys. Rev. B 2008, 77, 245408.

[34] L. G. Cançado, K. Takai, T. Enoki, M. Endo, Y. A. Kim, H. Mizusaki, A. Jorio, L. N. Coelho, R. Magalhães-Paniago, M. A. Pimenta, Appl. Phys. Lett. $2006,88,163106$

[35] H. Jussila, H. Yang, N. Granqvist, Z. Sun, Optica 2016, 3, 151-158.

[36] R. R. Nair, P. Blake, A. N. Grigorenko, K. S. Novoselov, T. J. Booth, T. Stauber, N. M. R. Peres, A. K. Geim, Science 2008, 320, 1308.

[37] B. D. Ratner, D. G. Castner, in Surf. Anal. Princ. Tech. (Ed.: I. S. G. John C. Vickerman), John Wiley \& Sons Ltd, Chichester, 2009, pp. 47-113.

[38] I. Cebula, H. Lu, M. Zharnikov, M. Buck, Chem. Sci. 2013, 4, 4455-4464.

[39] S. Ghosh, K. Ganesan, S. R. Polaki, T. R. Ravindran, N. G. Krishna, M. Kamruddin, A. K. Tyagi, J. Raman Spectrosc. 2014, 45, 642-649.

[40] T. Ciuk, S. Cakmakyapan, E. Ozbay, P. Caban, K. Grodecki, A. Krajewska, I. Pasternak, J. Szmidt, W. Strupinski, J. Appl. Phys. 2014, 116, 123708.

[41] X. Li, G. Zhang, X. Bai, X. Sun, X. Wang, E. Wang, H. Dai, Nat Nanotechnol. 2008, 3, 538-542.

[42] H. Medina, Y.-C. Lin, C. Jin, C.-C. Lu, C.-H. Yeh, K.-P. Huang, K. Suenaga, J. Robertson, P.-W. Chiu, Adv. Funct. Mater. 2012, 22, 2123-2128.

[43] X. Chen, Z. Chen, W. Jiang, C. Zhang, J. Sun, H. Wang, W. Xin, L. Lin, M. K. Priydarshi, H. Yang, Adv. Mater. 2017, 29, 1603428.

[44] D. Pech, M. Brunet, H. Durou, P. Huang, V. Mochalin, Y. Gogotsi, P.-L. Taberna, P. Simon, Nat. Nanotechnol. 2010, 5, 651.

Manuscript received: June 18, 2019

Revised manuscript received: August 2, 2019

Accepted manuscript online: August 6, 2019

Version of record online: August 25, 2019 